\title{
Strategic Research on the Fusion of Fashion Industry and New Media Industry in Wenzhou
}

\author{
Zhang De-Jia \\ Wenzhou Vocational \& Technical College, Wenzhou. 325035
}

Keywords: Wenzhou. Fashion Industry. New Media.Fusion.

\begin{abstract}
The fusion products of fashion industry and new media industry mainly include fashion blog and other fashion social media such as shopping website, online community, big data, ecommerce, electronic magazine, information website, fashion video website and so on. These new business products represent the fusion of the two industries. And they have gradually changed the consumption habits of people, enriched the diversity of fashion products and satisfied spiritual and cultural needs of the masses. What's more, these new products also have influence on the production mode of fashion enterprises and they have enhanced the productivity and organizational structure effectively through optimizing design, manufacturing and sale processes.
\end{abstract}

\section{Introduction}

New Media helps to lead the fashion trend and design direction through culture creativity, strengthen the productivity though technical innovation and change the consumption habits of people through convenient intelligent network terminal. Moreover, New Media reconstructs the traditional marketing mode of fashion industry by taking advantage of the interaction of cyber marketing. In addition, New Media's Right of Speech in the Fashion Industry has pointed out that new media is the important carrier of creativity in the fashion industry and it can also promote the consumption in the industry.

$\mathrm{Su}$, Chen zhongyu have discussed the social function of media in fashion communication. And they consider that the function has transformed from traditionally carrying fashion information to making, guiding, promoting and criticizing fashion. Besides, New Media has constantly changed the main dimensions of fashion communication, content reliability, channel width, terminal velocity and critical depth. Therefore, it will finally have influence on the entire process of fashion industry . They also point out that "Internet fashion communication has become the main mode of communication in the current fashion." Daixi Wu has discussed the transformation ways of traditional fashion media, including digitization, to innovate the fashion industry chain with collection resources, such as "media + community + e-commerce" mode. What's more, as far as Lizhe and Dujuan are concerned, fashion brands and sponsors (spokepersons) have more interaction on social media, focus more on images and the geographical position, and manage to combine offline activities of social media marketing. All those have become important channels in the process of network exploration, research and purchase "

\section{New Media have enhanced the development of fashion industry}

Eunju Ko, Carol M. Megehee has studied some fashion luxury brands and made the conclusion that Social media marketing activities can promote the appreciation of customer brand equity. Shuluan Li has studied the big data strategy of fashion industry and suggested that consumer's voice should be translated into numbers, and the online store should be the pre-test for the physical store, which will respond immediately and corrects big data.

Li shan and some other people analyzed fashion guide website and established an evaluation model of marketing effect of the website. They think the effect is to enhance the willingness of users to consulting products and further to buy the product and increase the influence and so on. In addition, we can observe the result through some kinds of indexes such as flow indicators, users' 
engagement, interface friendliness, sense of community and so on. Chang-Lin Yang, Rong-Hwa Huang studied online auction form, analyzed the influencing factors that influence the effect of online auction, and established the evaluation model based on those factors. They believe that the four key factors influencing online auction are network environment, consumers, learning innovation, and internal business mechanism.

\section{The development of cultural industry, the enhancement of Internet scale and the development of third-party payment technology have promoted the integration of fashion industry and new media industry.}

This article analyzed the reality and possibility of deep docking of the integration from Media and e-commerce cross boundary integration perspective. And we think that credibility should be enhanced through studying new media, changing the pattern, and making full use of new media resources. Besides, the fusion mechanism of fashion industry and new media industry is analyzed from the perspective of value chain, and the current integration of the two industries is analyzed based on its essence. On the basis of clarifying the fusion of fashion industry and new media , this article analyzed the effect of the fusion of two industries in detail and pointed out that the fusion is affecting the behavior of enterprises from multiple links in the value chain., so as to change the market structure and promote the competitiveness of the industry. Wenzhou is in a critical period of industry transformation and upgrading. And due to the combination of culture and the traditional manufacturing industry, the fashion industry has gradually become the momentum of economic development. Moreover, with the development of Internet technology, the new media industry now also plays a very important role in the development of the economy. Therefore, the research content of industry fusion is enriched theoretically.

\section{Theoretical Research}

\subsection{Industrial convergence}

In the study of the evolution of the American machine tool industry, Rosenberg(1963) pointed out that the application of similar production technologies in different industries will make the industry closely connected with the separation. Sahal believes that technological convergence is a process of technological innovation that has been widely applied and diffused in relevant industries. Yoffie(1997) believes that industry integration is "the integration of products that were originally independent after digital technology". Some scholars have made clear that the integration of industry is based on product. Meiyun Li(2005) pointed out that the industrial convergence is the process of becoming a direct competitor to each other after the industrial boundaries of two or more industries that were originally independent have been blurred. Industrial convergence is an innovative process in essence, and involves multiple levels of integration, including technology, business and management, products, enterprises and markets.

\subsection{Industrial symbiosis theory}

Industrial symbiosis was first developed in the economic field and it is a new concept that imitates natural ecosystem. The symbiosis refers to the material relation between the existence of the economic subject in the perspective of economics. The most widely accepted definition of industrial symbiosis is: Companies can jointly improve the viability and profitabilityof the enterprise through the cooperation between different enterprises. Meanwhile, resources can be saved and environment can be protected through this consensus. 


\subsection{The relationship theory between fashion industry and new media industry.}

In terms of fashion industry and new media industry relationship theory, fashion new media to fashion industry action theory, Yuan Lin pointed out that New Media helps to lead the fashion trend and design direction through culture creativity, strengthen the productivity though technical innovation. Su, Chen zhongyu have discussed the social function of media in fashion communication. And they consider that the function has transformed from traditionally carrying fashion information to making, guiding, promoting and criticizing fashion. Besides, New Media has constantly changed the main dimensions of fashion communication, content reliability, channel width, terminal velocity and critical depth. Therefore, it will finally have influence on the entire process of fashion industry. They also point out that "Internet fashion communication has become the main mode of communication in the current fashion." Daixi Wu has discussed the transformation ways of traditional fashion media, including digitization, to innovate the fashion industry chain with collection resources, such as "media + community + e-commerce" mode.

\section{The state-of-the-art fashion industry}

\subsection{Fashion industry comes from the upgrading of relevant traditional industries. Therefore, its culture is the core.}

After several decades of rapid economic development, the traditional garment industry and the industry of daily chemical products are now in a certain scale. Since the cultural attribute of fashion industry is mainly reflected in the design process, the value of the future design link of Wenzhou fashion industry will be the general trend.

\subsection{Relevant traditional industries are gradually upgrading to the fashion industry.}

From the supply side, China's fashion industry is undergoing the important stage of transformation and upgrading. Due to the shortage of land resources and the disappearance of demographic dividend, the production cost of enterprises has increased. The low price advantage of the past no longer exists, and production efficiency needs to be improved. From the demand side, the current fashion industry in China reflects the trend of fast fashion. The fast fashion of ZARA, HM, uniqlo and other brands has been highly praised and recognized by consumers. Unique product design, rich product category, satisfying consumption experience, deep brand connotation and a lot of other consumer demands have brought great challenges to enterprises.

\subsection{The development of new media industry}

The core of the media industry is content. And The new media industry realizes the combination of content and technology. The new media industry is developing rapidly, with increasing value and increasing influence. Moreover, The business model is constantly innovating and integrating with all kinds of traditional industries. As a result, the rapid development of new media has brought great impact on traditional media. In this context, the traditional media has been actively exploring the path of transformation and upgrading, and actively arranging the integration and development of the media.

\subsection{The fusion power of fashion industry and new media industry.}

The main driving factors of the integration of fashion industry and new media industry include internal factors and external factors. The internal factors include cultural commonness, demand power and supply power. While external factors include deregulation of government and diffusion of technological innovation. 


\section{Conclusion}

With the integration and development of Wenzhou's fashion industry and new media industry, both fashion enterprises and new media enterprises are faced with different levels of opportunities and challenges. Enterprises should clarify current industry development status and think about the future industry development trend, so as to improve competitiveness. With the deepening of the integration of the two industries, the industrial boundary willgradually blur and the market structure will also change. Therefore, clarifying the current situation and law of the industry is very important for government to make policy.

\section{References}

[1] Bing Kang, Successful Design of Print Ads and Graphic creativity, J.The Academic Journal of Nanjing University of the Arts (The Edition of Art and Design) 2015,(01):3-5.

[2] Mingfu Hu, The Trends and Reasons of Modern Packaging Individualized Design, J.Packaging Journal 2012, (07): 11-12

[3] Yongming Zhu, The Diagrams, Symbols and Languages in Visual Communication Design, J.The Academic Journal of Nanjing University of the Arts (The Edition of Art and Design) 2014, (01): 58-62

[4] Asoka. S., Fukuda, K. and Yamazaki, K. (2004) Effects of sleep-wake pattern and residential status on psychological distress in university students. Sleep and Biological Rhythms, 2 (3) :192198

[5] Fujino, Y., Iso, H., Inaba, Y., Koizumi, A., Kubo, T. for the Japanese Collaborative Cohort Study Group (2006) A Prospective Cohort Study of Shift Work and Risk of Ischemic Heart Disease in Japanese Male Workers. Am. J. Epidemiol., 164: 128 - 135

[6] Fiore, L., Fehr, D., Bodor, R., Drenner, A., Somasundaram, G., and Papanikolopoulos, N. "Multi-camera human activity monitoring," Journal of Intelligent and Robotic Systems, vol.52, no.1, pp.5-43, 2008.

[7] K.O.M. Doi, "Smartphone-based monitoring system for activities of daily living for elderly people and their relatives etc.," Proceedings of the 2013 ACM Conference on Pervasive and Ubiquitous Computing Adjunct Publication, UbiComp ’13 Adjunct, pp.103-106, ACM, 2013.

[8] L. Pei, R. Guinness, R. Chen, J. Liu, H. Kuusniemi, Y. Chen, L. Chen, and J. Kaistinen, “Human behavior cognition using smartphone sensors," Sensors, vol.13, no.2, pp.1402-1424, 2013.

[9] Kusano, K. Muro, H. Hayashi, T. Harada, F. and Shimakawa, H. "Derivation of life rhythm from tracing elderly movement," The 10th Forum on Information Technology, FIT2011, pp.891892, 2011 